\title{
Blood-brain barrier transcytosis genes, risk of dementia and stroke: a prospective cohort study of 74,754 individuals
}

\author{
Ida Juul Rasmussen ${ }^{1,2} \cdot$ Anne Tybjærg-Hansen ${ }^{1,2,3,4} \cdot$ Katrine Laura Rasmussen ${ }^{1,2} \cdot$ Børge G. Nordestgaard ${ }^{2,3,4,5}$. \\ Ruth Frikke-Schmidt ${ }^{1,2,4}$
}

Received: 29 June 2018 / Accepted: 16 February 2019 / Published online: 4 March 2019

(C) The Author(s) 2019

\begin{abstract}
To test whether genetic variants in PICALM, BIN1, CD2AP, and RIN3-suggested to be involved in blood-brain barrier amyloid- $\beta$ transcytosis pathways - associate with Alzheimer's disease, all dementia, suggested vascular dementia, and stroke, and whether such associations are independent of the strong $84 A P O E$ risk allele. In a prospective cohort study of 74,754 individuals from the general population we genotyped PICALM (rs10792832), BIN1 (rs6733839), CD2AP (rs10948363), and RIN3 (rs10498633), and generated a weighted and a simple allele score. Multifactorially adjusted hazard ratios for the fourth quartile versus the first quartile of the weighted allele score were 1.42 (95\% confidence interval 1.22-1.64) for Alzheimer's disease, and 1.33 (1.19-1.48) for all dementia. For suggested vascular dementia and stroke the corresponding estimates were 1.71 (1.18-2.49) and 1.12 (1.04-1.22), respectively. Hazard ratios were similar after APOE adjustment. Genetic variants in PICALM, BIN1, CD2AP, and RIN3 are associated with increased risk of Alzheimer's disease, all dementia, and suggested vascular dementia independent of the strong $A P O E \varepsilon 4$ allele. These findings may suggest that clathrin-mediated endocytosis in clearance of amyloid- $\beta$ across the blood-brain barrier is important for the integrity of both brain tissue and cerebral vessels.
\end{abstract}

Keywords Alzheimer's disease $\cdot$ Vascular dementia $\cdot$ Amyloid- $\beta \cdot$ Epidemiology $\cdot$ Stroke $\cdot$ Blood-brain barrier clearance

\section{Introduction}

Alzheimer's disease and other forms of dementia are devastating neurodegenerative diseases currently affecting more than 47 million people globally, and expected to triple in 2050 [1]. There are no available curative treatments, no early preclinical, easily accessible biomarkers [2, 3], and large parts of the underlying biology remain unknown. Clinically, Alzheimer's disease often coexists with cerebral vascular diseases [4-8], and the major pathological hallmark of Alzheimer's disease is accumulation of a neurotoxic, sticky peptide, amyloid- $\beta$, in the brain and in cerebral vessels [3, 9-12]. An important clearance pathway of amyloid- $\beta$ from the brain is via transcytosis across the blood-brain barrier into the vascular lumen [13-15].

Interestingly, a number of genome-wide association studies (GWAS) risk genes [16], PICALM (phosphatidylinositolbinding clathrin assembly protein) $[17,18], B I N 1$ (bridging integrator 1) [19-21], CD2AP (CD2-associated protein) [22, 23], and RIN3 (Ras and Rab interactor 3) [24], encode proteins that are either directly or indirectly involved in transcytosis of amyloid- $\beta$ across the blood-brain barrier [24-32].

5 Department of Clinical Biochemistry, Herlev and Gentofte Hospital, Herlev Ringvej 75, 2730 Herlev, Denmark 
Particularly genetic variants tagging PICALM consistently associate with dementia in GWAS [16, 30, 33-35], and this signal is one of the most consistent after the $\varepsilon 4$ allele of APOE (apolipoprotein E gene) [29], an association first recognized in 1993 [36] and since validated globally [2, 3, 16, $30,31,35,37]$. PICALM is important for endocytosis and internalization of cell receptors, and is involved in clathrinmediated endocytosis $[28,38]$. Recently it was shown that PICALM regulates amyloid- $\beta$ blood-brain barrier transcytosis and clearance by initiating clathrin-mediated endocytosis via interaction with LRP1 (low-density lipoprotein receptor related protein-1). LRP1 is a key amyloid- $\beta$ clearance acceptor that also binds to apoE [39].

Our aim was dual. First, we wanted to establish the exact risk increases at the individual level for Alzheimer's disease and all dementia for four potential blood-brain barrier pathway genes. Second, we wanted to test the impact on specific cerebral vascular endpoints -suggested vascular dementia and stroke-since the blood-brain barrier most likely is central for dementia related vascular events. We genotyped four variants in PICALM, BIN1, CD2AP, and RIN3 previously identified as top hits for Alzheimer's disease [16], in two prospective studies of the general population totaling 74,754 individuals.

\section{Materials and methods}

\section{Participants}

The Copenhagen General Population Study (CGPS) is a prospective study of the Danish general population initiated in 2003 and still recruiting [40-42]. Individuals were selected randomly based on the national Danish Civil Registration System to reflect the adult Danish population aged $20-80+$ years. Data were obtained from a self-administered questionnaire reviewed together with an investigator at the day of attendance, a physical examination, and from blood samples including DNA extraction. Genotypes are available on 64,974 individuals. Before genotyping all DNA samples were blinded regarding phenotype and endpoint to the laboratory technician and investigator.

The Copenhagen City Heart Study (CCHS) is a prospective study of the Danish general population initiated in 1976-1978 with follow-up examinations in 1981-1983, 1991-1994 and 2001-2003 [40-42]. Individuals were recruited and examined exactly as in the CGPS. Genotypes are available on 9780 individuals from the 1991-1994 and 2001-2003 examinations.

Combining the two studies yielded a total of 74,754 individuals for Alzheimer's disease and all dementia analyses, of whom 2514 developed dementia during a median follow-up of 10 years (range 0-23 years). For the analyses of suggested vascular dementia and stroke we included 72,612 individuals, of whom 5016 developed stroke and 248 developed suggested vascular dementia during a median follow-up of 10 years (range 0-25 years). No individuals were lost to follow-up. Follow-up began at the time of blood sampling (2003 and onward for CGPS and 1991-1994 or 2001-2003 for CCHS). Follow-up ended at occurrence of event ( $\mathrm{n}=2514$ for Alzheimer's disease and all dementia and $\mathrm{n}=5264$ for suggested vascular dementia and stroke), death $(n=10,608$ for Alzheimer's disease and all dementia and $n=9203$ for suggested vascular dementia and stroke), emigration ( $n=404$ for Alzheimer's disease and all dementia and $n=395$ for suggested vascular dementia and stroke), or on March 22nd, 2017 (last update of the registry), whichever came first. Years at risk were calculated for each participant as the time difference between baseline and the end of follow-up. Written informed consent was obtained from all individuals. Individuals in both studies were white and of Danish descent.

\section{Dementia and stroke}

In CGPS and CCHS, information on births, deaths, emigrations and immigrations was collected from the national Danish Civil Registration System. Information on diagnoses of dementia and stroke, and age at diagnosis was drawn from the national Danish Patient Registry and the national Danish Causes of Death Registry. The national Danish Patient Registry has information on all patient contacts with all clinical hospital departments in Denmark since 1977, including emergency wards and outpatient clinics from 1994. The national Danish Causes of Death Registry contains data on the causes of all deaths in Denmark, as reported by hospitals and general practitioners. The national Danish registries are regarded among the best of its kind [43, 44], however an inherent limitation of using registrybased data is underdiagnosis, because only hospital registered events are in the registries. This is a general issue that needs to be considered when interpreting results based on registry diagnoses. In Denmark the diagnosis of dementia has been made in accordance with international standards in routine clinical practice since the 1990's and 91-95\% of diagnoses are given by neurologically relevant units and/or departments of internal medicine [45]. Alzheimer's disease is diagnosed using the NINCDS-ADRDA criteria [46], the NIA-AA criteria [47], or the ICD8/ICD10 criteria and is highly valid once the diagnosis is given [48]. Alzheimer's disease was ICD8 (World Health Organization International Classification of Diseases, 8th revision) code 290.10 and ICD10 (World Health Organization International Classification of Diseases, 10th revision) codes F00 and G30. Vascular dementia has been diagnosed using the ICD10 criteria, the NINDS-AIREN criteria (before 2015) [49] or the VASCOG 
criteria since 2015 [50]. The diagnosis, however, suffer from some uncertainty [48], which is why we use the term "suggested vascular dementia" throughout the paper. The diagnosis of suggested vascular dementia was ICD10 code F01 and did not include mixed dementia. All dementia further included unspecified dementia (ICD8 290.18; ICD10 F03). Stroke was ICD8 codes 430, 431 and 433-435 and ICD10 code G45, I60, I61, I63 and I64.

\section{Laboratory analyses}

Standard hospital assays measured electrolytes, glucose, liver-, kidney-, and inflammatory parameters as well as total cholesterol, HDL (high-density lipoprotein) cholesterol and triglycerides (Boehringer Mannheim, Mannheim, Germany). LDL (low-density lipoprotein) cholesterol was calculated using the Friedewald equation [51] when plasma triglycerides were $\leq 4 \mathrm{mmol} / \mathrm{L}(\leq 352 \mathrm{mg} / \mathrm{dL})$, and otherwise measured directly (Konelab). Estimated glomerular filtration rate was calculated according to CKD-EPIcrea [52].

\section{Genotyping}

Taqman-based (Life Technologies, a part of Thermo Fisher Scientific, Waltham, Massachusetts, USA) or KASP technology based assays (LGC Genomics, Hoddesdon, Herts, UK) were used to genotype for PICALM rs10792832, BIN1 rs6733839, CD2AP rs10948363, RIN3 rs10498633 and for p.Cys130Arg (rs429358, legacy name Cys112Arg, c.388T $>$ C) defining the $\varepsilon 4$ allele and p.Arg 176Cys (rs7412, legacy name $\operatorname{Arg} 158 \mathrm{Cys}, \mathrm{c} .526 \mathrm{C}>\mathrm{T}$ ) defining the $\varepsilon 2$ allele of the $A P O E$ gene [42].

\section{Other covariates}

Body mass index was measured weight in kilograms divided by measured height in meters squared. Hypertension was use of anti-hypertensive medication, a systolic blood pressure of $140 \mathrm{~mm} \mathrm{Hg}$ or greater, and/or a diastolic blood pressure of $90 \mathrm{~mm} \mathrm{Hg}$ or greater. Diabetes mellitus was self-reported disease, use of insulin or oral hypoglycemic agents, and/or non-fasting plasma glucose levels of more than $11 \mathrm{mmol} / \mathrm{L}$ $(>198 \mathrm{mg} / \mathrm{dL}$ ). Estimated glomerular filtration rate was calculated according to CKD-EPIcrea [52]. Smoking was current smoking. High alcohol consumption was $>14 / 21$ units per week for women/men ( 1 unit $=12 \mathrm{~g}$ alcohol, equivalent to one glass of wine or one beer ( $33 \mathrm{cL})$ ). Physical inactivity was $\leq 4 \mathrm{~h}$ per week of light physical activity in leisure time. Women reported menopausal status and use of hormonal replacement therapy. Lipid-lowering therapy was mainly statins (yes/no), and low education was $<8$ years of education.

\section{Consortia data}

IGAP (the International Genomics of Alzheimer's Project) (is a large two-stage study based upon GWAS on individuals of European ancestry [16]. In stage 1, IGAP used genotyped and imputed data on 7,055,881 SNPs (single nucleotide polymorphisms) to meta-analyze four previously published GWAS datasets consisting of 17,008 Alzheimer's disease cases and 37,154 controls (The European Alzheimer's disease Initiative-EADI, the Alzheimer Disease Genetics Consortium-ADGC, the Cohorts for Heart and Aging Research in Genomic Epidemiology consortium-CHARGE, the Genetic and Environmental Risk in AD consortium-GERAD). In stage 2, 11,632 SNPs were genotyped and tested for association in an independent set of 8572 Alzheimer's disease cases and 11,312 controls. Finally, a meta-analysis was performed combining results from stages 1 and 2 [16].

\section{Statistical analysis}

We used Stata/S.E. v14.0 and v13.0 (Stata Corp, College Station, TX, USA). Probability values $<0.001$ are given as powers of 10. Kruskal-Wallis one-way analysis of variance or Pearson's $\chi^{2}$ test were used to evaluate continuous and categorical variables by genotype and disease status. Missing data on continuous covariates were imputed from age, sex, and the most related continuous parameters. Missing data on categorical covariates were assigned a dummy value. Missing values for continuous covariates were $<0.8 \%$.

Combining all genotypes, we generated two different genetic risk scores for dementia and stroke. The first genetic score, named "weighted allele score", was calculated for each individual using a weighted sum of alleles for increasing risk of Alzheimer's disease and all dementia, subsequently categorized into quartiles of approximately equal size to maximize statistical power. The weights correspond to the regression coefficients for the dementia risk increasing alleles in each individual adjusted for age and gender [53] (Supplementary Table 1). The weights were generated in the combined cohort. The second genetic score, named "simple allele score", was a simple counting of the number of dementia increasing risk alleles in each individual, subsequently categorized into three groups of approximately equal size. Similar scores were generated for suggested vascular dementia and stroke.

Cumulative incidences of Alzheimer's disease and all dementia were plotted against age and weighted/simple allele score group, using the method of Fine-Gray [54], to account for the possibility of death as a competing event. Similar cause-specific (censoring at death) Cox proportional hazards regression models with age as time scale and left truncation (delayed entry) were used to estimate hazard 
ratios for Alzheimer's disease, all dementia, suggested vascular dementia, and stroke as a function of weighted allele score and simple allele score. Using age as time scale ensures that each participant experiencing an event is always compared to a participant at the exact same age. This is regarded as the state of the art age-adjustment method in survival analyses in large epidemiological studies where participants enter at different ages. For Cox regression models, proportionality of hazards over time were assessed by plotting $-\ln (-\ln$ [survival] $)$ versus $\ln$ (analysis time). There was no suspicion of nonproportionality. Cox regression models were multifactorially adjusted for age (as time scale), sex, body mass index, hypertension, diabetes, smoking, alcohol intake, physical inactivity, postmenopausal status and hormonal replacement therapy in women, lipid-lowering therapy, educational level, and APOE genotype ( $22 / \varepsilon 3 / \varepsilon 4$ APOE genotype).

Meta-analyses were conducted using the user-written metan command from Stata/S.E. v13.0 to estimate fixed and random effects odds ratios by regression coefficients and standard errors for each of the four genetic variants. Between-study heterogeneity was assessed by Cochran's Q test and $\mathrm{I}^{2}$ statistics and was considered as low $\left(\mathrm{I}^{2} \leq 25 \%\right)$, moderate $\left(25 \%<\mathrm{I}^{2}<49 \%\right)$, or high $\left(\mathrm{I}^{2} \geq 50 \%\right)$. Results are presented for both fixed-and random-effects models.

\section{Results}

Baseline characteristics of the 74,754 individuals enrolled in the study are shown by quartiles of weighted allele score for Alzheimer's disease in Table 1. Baseline characteristics by disease status are shown in Supplementary Table 2 . Age at diagnosis of disease are shown in Supplementary Table 3 and distribution of age at baseline and follow-up time are shown in density plots in Supplementary Figures 1 and 2. All results are for the CGPS and CCHS combined unless otherwise stated.
Table 1 Characteristics of study participants by weighted allele score quartile for Alzheimer's disease

\begin{tabular}{|c|c|c|c|c|}
\hline & 1st quartile & 2nd quartile & 3rd quartile & 4th quartile \\
\hline No. of individuals (\%) & $19,423(26)$ & $18,251(24)$ & $18,409(25)$ & $18,671(25)$ \\
\hline Age (years) & $58(48-67)$ & $58(48-67)$ & $58(47-67)$ & $57(47-67)$ \\
\hline Female (\%) & 55 & 56 & 55 & 56 \\
\hline Total cholesterol $(\mathrm{mmol} / \mathrm{L})$ & $5.6(4.9-6.4)$ & $5.6(4.9-6.4)$ & $5.6(4.9-6.4)$ & $5.6(4.9-6.4)$ \\
\hline LDL cholesterol (mmol/L) & $3.3(2.6-3.9)$ & $3.2(2.6-3.9)$ & $3.2(2.6-3.9)$ & $3.3(2.6-3.9)$ \\
\hline HDL cholesterol (mmol/L) & $1.5(1.2-1.9)$ & $1.6(1.3-1.9)$ & $1.5(1.2-1.9)$ & $1.6(1.2-1.9)$ \\
\hline Triglycerides (mmol/L) & $1.4(1.0-2.1)$ & $1.4(1.0-2.1)$ & $1.4(1.0-2.1)$ & $1.4(1.0-2.1)$ \\
\hline Body mass index $\left(\mathrm{kg} / \mathrm{m}^{2}\right)$ & $26(23-28)$ & $26(23-28)$ & $26(23-28)$ & $26(23-28)$ \\
\hline Hypertension (\%) & 58 & 59 & 58 & 58 \\
\hline Diabetes mellitus (\%) & 4 & 4 & 4 & 4 \\
\hline Smoking (\%) & 23 & 24 & 24 & 24 \\
\hline High alcohol consumption (\%) & 18 & 18 & 18 & 18 \\
\hline Physical inactivity (\%) & 53 & 52 & 53 & 52 \\
\hline Postmenopausal (\%) ${ }^{\mathrm{a}}$ & 66 & 66 & 67 & 67 \\
\hline Hormonal replacement therapy $(\%)^{\mathrm{a}}$ & 11 & 12 & 11 & 11 \\
\hline Lipid-lowering therapy (\%) & 9 & 10 & 9 & 9 \\
\hline Education $<8$ years $(\%)$ & 14 & 14 & 14 & 15 \\
\hline
\end{tabular}

Values are median (interquartile range) or percent and are from the day of enrolment (2003 and onwards for the CGPS and 1991-1994 or 2001-2003 for the CCHS). Hypertension was use of anti-hypertensive medication, a systolic blood pressure of $140 \mathrm{~mm} \mathrm{Hg}$ or greater, and/or a diastolic blood pressure of $90 \mathrm{~mm} \mathrm{Hg}$ or greater. Diabetes mellitus was self-reported disease, use of insulin or oral hypoglycemic agents, and/or non-fasting plasma glucose levels of more than $11 \mathrm{mmol} / \mathrm{L}$ (>198 mg/dL). Smoking was current smoking. High alcohol consumption was $>14 / 21$ units per week for women/men ( 1 unit $=12 \mathrm{~g}$ alcohol, equivalent to one glass of wine or one beer $(33 \mathrm{cL})$ ). Physical inactivity was $\leq 4 \mathrm{~h}$ per week of light physical activity in leisure time. Women reported menopausal status and use of hormonal replacement therapy. Lipid-lowering therapy was primarily statins (yes/no), and low education was $<8$ years of education. Differences across weighted allele score groups were tested by Kruskal-Wallis one-way analysis of variance or Pearson's $\chi^{2}$ test

$H D L$ high-density lipoprotein cholesterol, $L D L$ low-density lipoprotein cholesterol

${ }^{\mathrm{a}}$ In women only 


\section{Weighted/simple allele scores and risk of Alzheimer's disease and all dementia}

Cumulative incidences of Alzheimer's disease and all dementia increased stepwise as a function of increasing weighted/simple allele score groups (all $P$ for trend $\leq 2 \times 10^{-5}$ ) (Fig. 1). Multifactorially adjusted hazard ratios for the fourth versus the first weighted allele score quartile were 1.42 (95\% confidence interval 1.22-1.64) for Alzheimer's disease and 1.33 (1.19-1.48) for all dementia. For the simple allele score multifactorially adjusted hazard ratios for 5-8 alleles versus $0-3$ alleles were 1.32 (1.16-1.51) for Alzheimer's disease and 1.26 (1.14-1.38) for all dementia (Fig. 2, left panel). Results were similar after further adjustment for APOE genotype (Fig. 2, middle panel), or when analyses were performed exclusively in $A P O E \varepsilon 33$ carriers (Fig. 2, right panel). Findings for Alzheimer's disease were comparable to results using external weights (Supplementary Figure 3) [16]; external weights were not available for all dementia. Findings were similar for the CGPS and CCHS separately (Supplementary

\section{Alzheimer's disease}
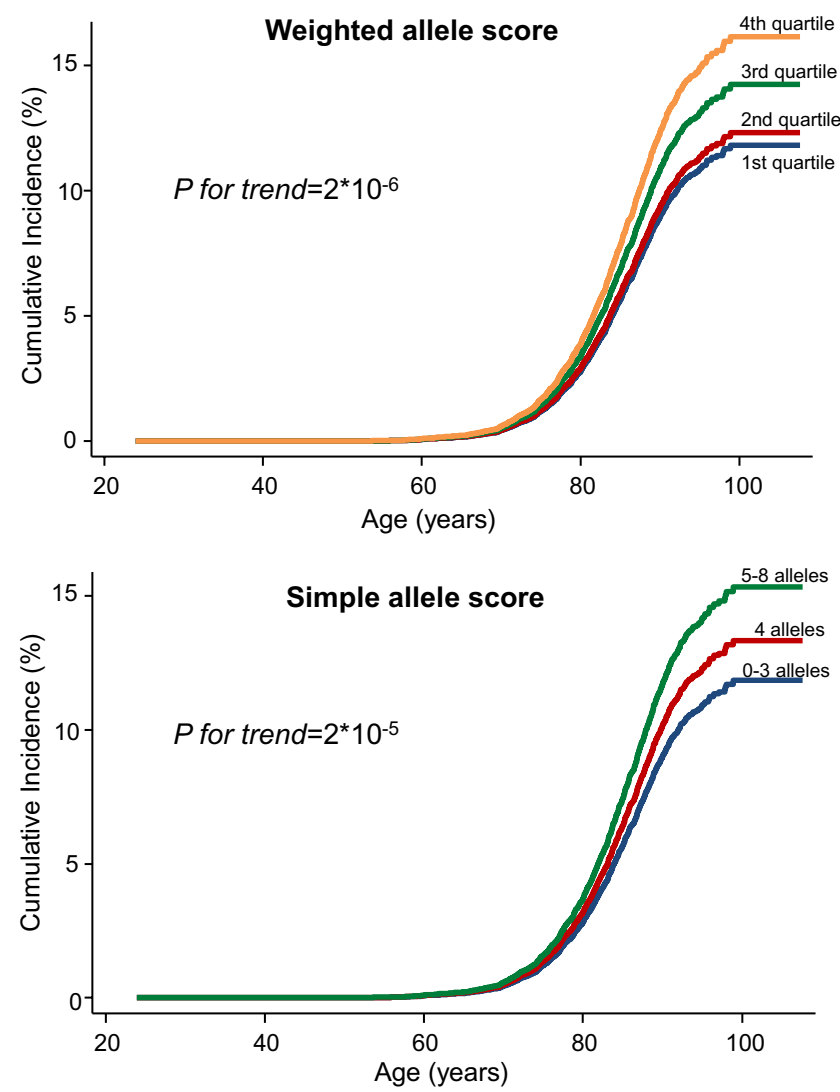

Fig. 1 Cumulative incidence of Alzheimer's disease and all dementia by age and weighted/simple allele scores. We used Fine-Gray models, allowing for death as a competing event. $P$ for trend from competing
Figures 4 and 5) and when excluding participants who developed dementia of any subtype within the first six months of follow-up (Supplementary Figure 6). When dividing the weighted allele score into tertiles, hazard ratios for the third tertile were similar to hazard ratios for the fourth quartile in the original analysis for both Alzheimer's disease and all dementia (Supplementary Figure 7). We also performed the analysis in APOE \&4-carriers and $\varepsilon 4$-non-carriers separately and results were similar (Supplementary Figure 8). After stratification in age groups ( $<65$ years, $65-80$ years and $\geq 80$ years), results were similar although attenuated in the $\geq 80$ years age group, most likely due to lack of power (Supplementary Figure 9). There was no interaction between the genetic scores and age at baseline (Alzheimer's disease: $P=0.43$ for the weighted allele score and $P=0.61$ for the simple allele score. All dementia: $P=0.53$ for the weighted allele score and $P=0.18$ for the simple allele score). Further no interaction was present between the genetic scores and $A P O E$ genotype (Alzheimer's disease: $P=0.31$ for the weighted allele score and $P=0.52$ for the simple allele
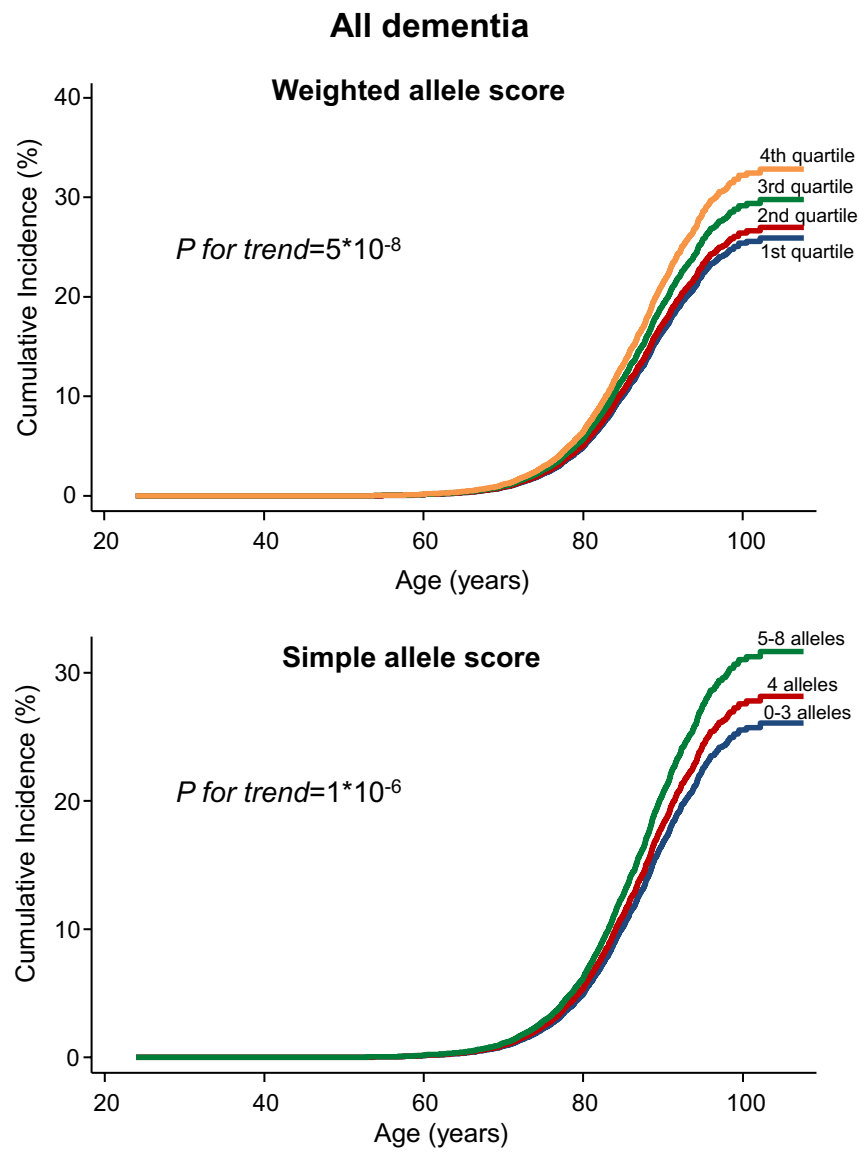

risks regression trend test. Weights for the weighted allele score were generated in the combined cohort 


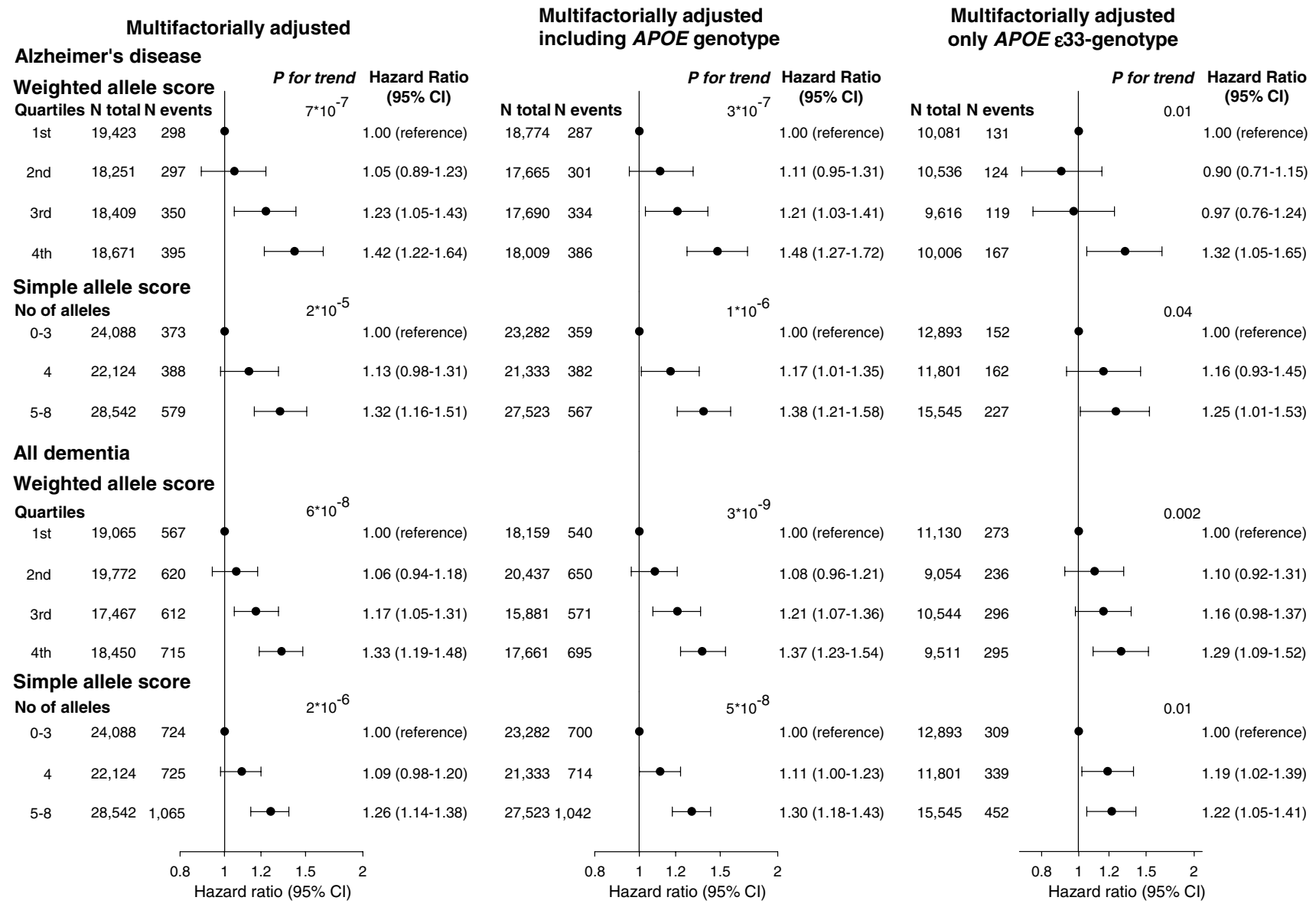

Fig. 2 Risk of Alzheimer's disease and all dementia as a function of weighted/simple allele scores. Individuals with Alzheimer's disease or all dementia before blood sampling were excluded, leaving 74,754 individuals for analysis in the left panel. A total of 72,138 individuals with available $A P O E$ genotype were included in the middle panel. The right panel exclusively contains individuals with the APOE $\varepsilon 33$ genotype $(\mathrm{N}=40,239)$. Hazard ratios were multifactorially adjusted

score. All dementia: $P=0.91$ for the weighted allele score and $P=0.22$ for the simple allele score).

\section{Meta-analyses of individual genetic variants for dementia}

Meta-analyses included the present two prospective cohorts of the Danish general population and stage 1 and 2 from IGAP which includes meta-analyzed data from 15 individual studies [16]. For PICALM overall fixed- and random-effects odds ratios were $1.14(1.12-1.17)$ and $1.14(1.11-1.17)$ with $I^{2}$ of $20 \%$ ( $P$ for heterogeneity $=0.29$ ) (Fig. 3, upper left panel). For BINl odds ratios were $1.21(1.19-1.24)$ for both overall fixed- and random-effects, with $I^{2}$ of $0 \%$ ( $P$ for heterogeneity $=0.97)($ Fig. 3, upper right panel). For $C D 2 A P$ odds ratios were $1.10(1.08-1.13)$ for both overall fixed- and random-effects, with $I^{2}$ of $0 \%(P$ for heterogeneity $=0.92)$ for age (as time scale), sex, hypertension, diabetes, smoking, alcohol intake, physical inactivity, postmenopausal status and hormonal replacement therapy in women, lipid-lowering therapy and educational level (left and right panel). Middle panel additionally includes adjustment for $A P O E$ genotype. $P$ for trend from Cox regression. $A P O E=$ apolipoprotein $\mathrm{E}$ gene; $A P O E$ genotype $=\varepsilon 2 / \varepsilon 3 / \varepsilon 4 A P O E$ genotype; $\mathrm{CI}=$ confidence interval

(Fig. 3, lower left panel). For RIN3 odds ratios were 1.11 (1.08-1.13) for both overall fixed- and random-effects, with $I^{2}$ of $0 \%(P$ for heterogeneity $=0.70)$ (Fig. 3, lower right panel).

\section{Weighted/simple allele scores and risk of suggested vascular dementia and stroke}

Multifactorially adjusted hazard ratios for the fourth versus the first weighted allele score quartile were $1.71(1.18-2.49)$ for suggested vascular dementia and 1.12 (1.04-1.22) for stroke. When prevalent stroke was excluded, risk of suggested vascular dementia remained (fourth versus first quartile 2.01 (1.32-3.07), data not shown). For the simple allele score multifactorially adjusted hazard ratios for 5-8 alleles versus $0-3$ alleles were $1.65(1.20-2.26)$ for suggested vascular dementia and $1.03(0.96-1.10)$ for stroke (Fig. 4, left 


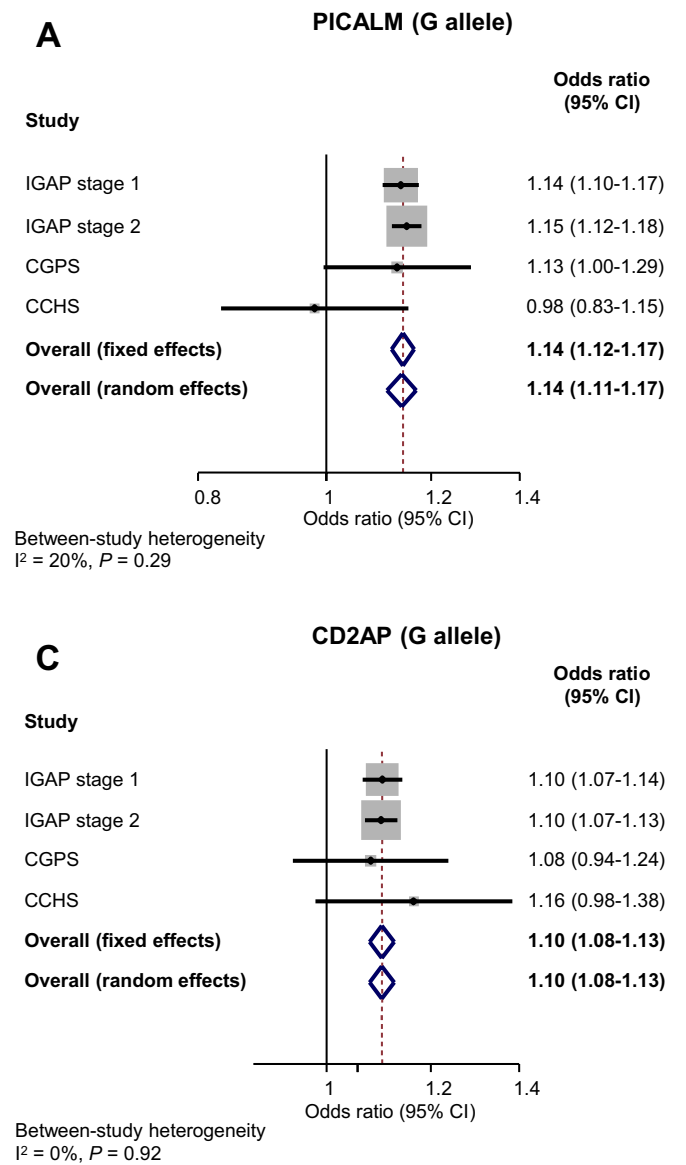

Fig. 3 Meta-analysis of PICALM, BIN1, CD2AP, and RIN3 in IGAP 1 and 2, CGPS, and CCHS. Summarizing risk of Alzheimer's disease by risk increasing alleles in PICALM (G allele), BIN1 (T allele), $C D 2 A P$ (G allele), and RIN3 (G allele). Horizontal lines correspond to $95 \%$ confidence intervals by forest plots. Diamonds and broken vertical lines represent summary estimates. Confidence intervals for the summary estimates correspond to the width of the diamonds. Grey shaded areas correspond to the weights of the studies in the

panel). Results were similar after further adjustment for $A P O E$ genotype (Fig. 4, right panel), and when excluding participants who developed dementia of any subtype within the first six months of follow-up (Supplementary Figure 10).

\section{Blood pressure, heart rate and biochemical characteristics of study participants by weighted allele score}

Weighted allele score quartiles for all endpoints, were not associated with any variation in vital signs (systolic and diastolic blood pressure, heart rate), plasma levels of electrolytes (potassium, sodium, chloride), renal function (creatinine, estimated glomerular filtration rate), liver function (alanine aminotransferase, alkaline phosphatase, bilirubin, gamma-glutamyl transpeptidase), glucose level or highsensitivity C-reactive protein (Supplementary Tables 4 and
B

BIN1 (T allele)
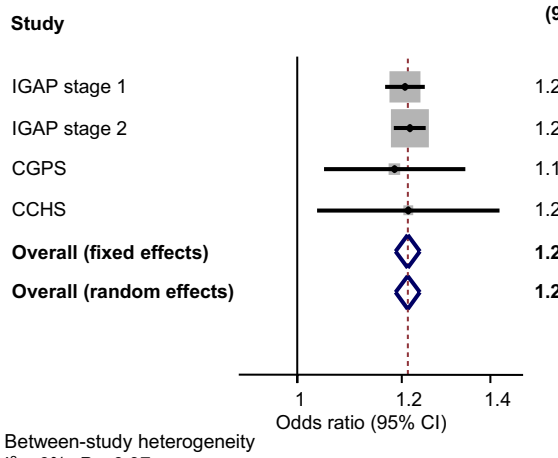

Odds ratio $(95 \% \mathrm{Cl})$

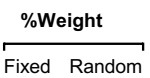

$1.21(1.17-1.25) \quad 37 \quad 37$

$1.22(1.18-1.25) \quad 58 \quad 58$

$1.19(1.05-1.34) \quad 3 \quad 3$

$1.21(1.04-1.42) \quad 2 \quad 2$

$1.21(1.19-1.24)$

$.21(1.19-1.24)$

Between-study heterogeneity $1^{2}=0 \%, P=0.97$

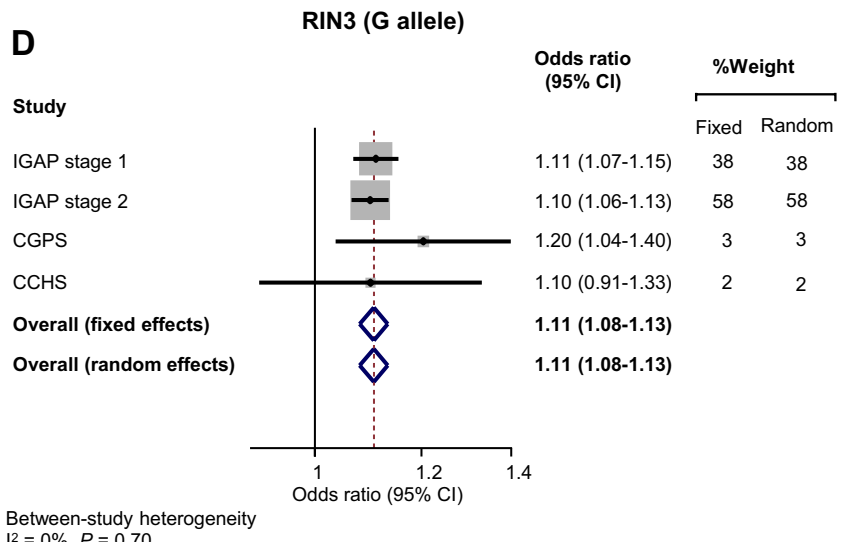
$1^{2}=0 \%, P=0.70$

meta-analysis from the fixed effects models (right column). $P$ for heterogeneity from Cochran's $\mathrm{Q}$ test. $A P O E=$ apolipoprotein $\mathrm{E}$ gene; $A P O E$ genotype $=\varepsilon 2 / \varepsilon 3 / \varepsilon 4 A P O E$ genotype $B I N 1=$ bridging integrator 1 ; $\mathrm{CCHS}=$ the Copenhagen City Heart Study; $C D 2 A P=\mathrm{CD} 2$ associated protein; $\mathrm{CGPS}=$ the Copenhagen General Population Study; $\mathrm{CI}=$ confidence interval; IGAP $=$ the International Genomics of Alzheimer's Project; PICALM=phosphatidylinositol-binding clathrin assembly protein; RIN3= Ras and Rab interactor 3

5 for Alzheimer's disease and all dementia; data not shown for suggested vascular dementia and stroke).

\section{Discussion}

The principal findings of this study are that genetic variation in PICALM, BIN1, CD2AP and RIN3, four genes suggested to be involved in blood-brain barrier amyloid- $\beta$ transcytosis, are associated with increased risk of Alzheimer's disease, all dementia, and suggested vascular dementia in the general population independent of the strong $A P O E$ genotype. There was no conclusive association with risk of stroke. These findings may suggest that compromising blood-brain barrier function has implications for both brain tissue-manifested by increased Alzheimer's disease risk—as well as for 


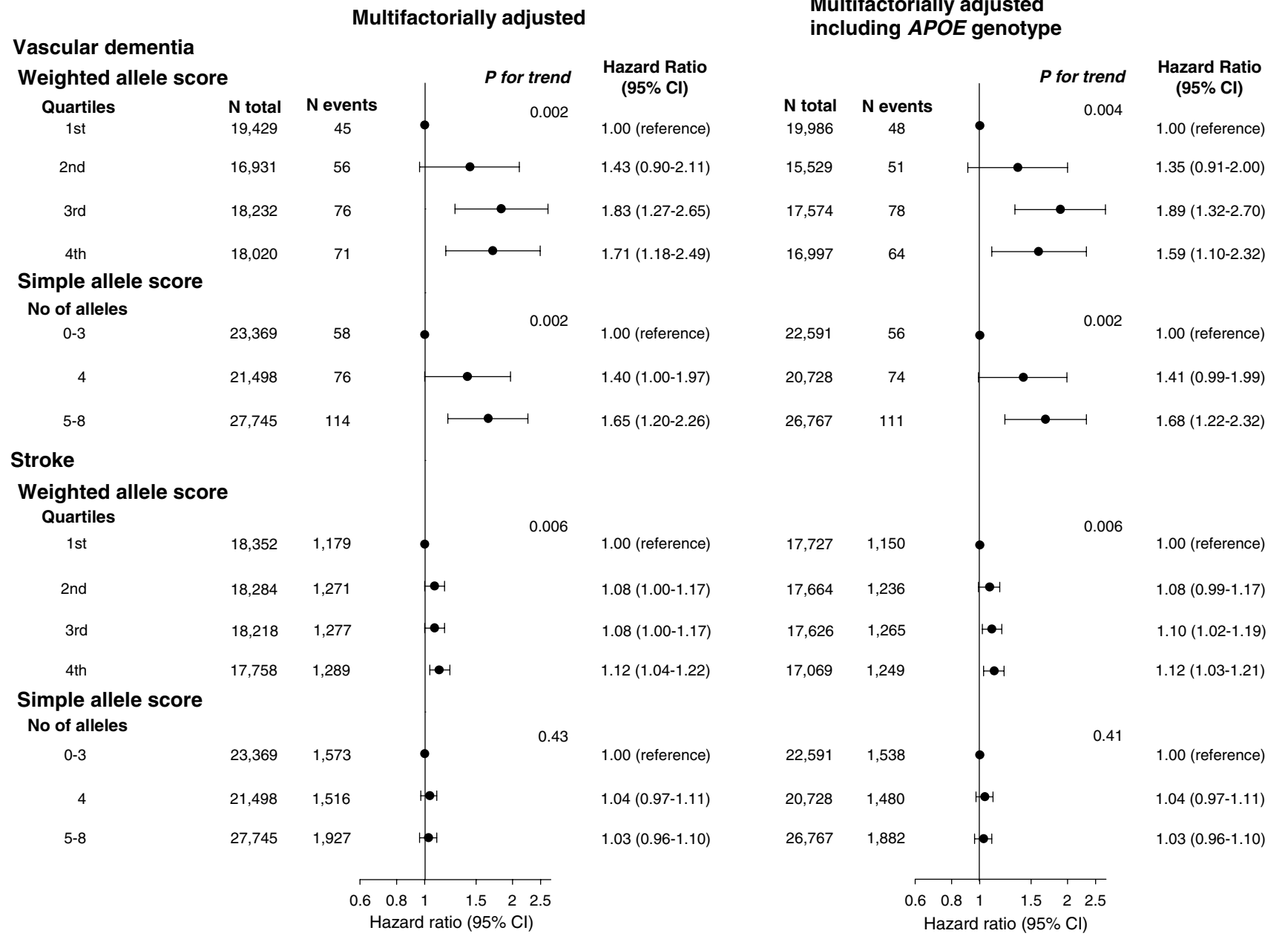

Fig. 4 Risk of suggested vascular dementia and stroke as a function of weighted/simple allele scores. Individuals with suggested vascular dementia or stroke before blood sampling were excluded, leaving 72,612 individuals for analysis in the left panel. A total of 70,086 individuals with available $A P O E$ genotype were included in the right panel. Hazard ratios were multifactorially adjusted for age (as time scale), sex, hypertension, diabetes, smoking, alcohol intake, physical inactivity, postmenopausal status and hormonal replacement therapy in women, lipid-lowering therapy and educational level. Right panel additionally includes adjustment for $A P O E$ genotype. $P$ for trend from Cox regression. $A P O E=$ apolipoprotein E gene; $A P O E$ genotype $=\varepsilon 2 / \varepsilon 3 / \varepsilon 4 A P O E$ genotype $; \mathrm{CI}=$ confidence interval cerebral vasculature-manifested by increased risk of suggested vascular dementia.

To our knowledge this is the first study to simultaneously assess risk of dementia and stroke as a function of genetic variation in all four, potential blood-brain barrier transcytosis genes. PICALM has consistently been associated with Alzheimer's disease in several GWA studies with risk estimates in the range of 1.14-1.27 [16, 30, 33, 34]. BIN1 in the range of $1.15-1.36$ [16, 31, 33-35, 37], CD2AP in the range of 1.10-1.12 [16, 31, 37], and finally, RIN3 with an odds ratio for the risk increasing allele of 1.10 [16]. The present findings of these genes with suggested vascular dementia are however novel.

The biological mechanisms of our findings remain to be determined and the evidence from population studies linking the four selected variants to blood-brain barrier amyloid- $\beta$ transcytosis pathways is limited. Recently new light was shed on the function of PICALM in Alzheimer's disease pathology, emphasizing clathrin-mediated endocytosis as a potential important mechanism in amyloid- $\beta$ clearance across the blood-brain barrier [29]. Zhao et al. showed that reductions of PICALM in brain endothelium in Alzheimer's disease correlated with amyloid- $\beta$ accumulation, Alzheimer's disease neuropathology, and cognitive impairment in mice. Picalm ${ }^{+/-}$mice had a reduction in amyloid- $\beta 40$ and amyloid- $\beta 42$ efflux across the blood-brain barrier of $41 \%$ and $61 \%$, respectively, compared to littermate controls. Using in vitro endothelial monolayer to mimic the blood-brain barrier, they found that amyloid- $\beta$ binding to LRP1 enhanced the binding of PICALM, which initiated PICALM/clathrin-dependent endocytosis of the amyloid$\beta$-LRP1 complex and subsequent transcytosis involving 
GTPases Rab5 and Rab11 controlling, respectively, early endosome formation and vesicle exocytosis [55-60]. In primarily in vitro studies both BIN1 and CD2AP are shown to bind to Rab5 [27], and RIN3 is shown to mediate the recruitment of BIN1 and CD2AP to Rab5-positive early endosomes in the endocytic transport pathway [24, 27]. Taken together, these findings suggest that PICALM and interacting proteins control amyloid- $\beta$ transport across the blood-brain barrier and clearance of amyloid- $\beta$ from brain through clathrinmediated endocytosis. Therefore, it may be biologically plausible that inactivating genetic variants in genes involved in this transcytosis pathway may cause an increased risk of Alzheimer's disease due to accumulation of amyloid- $\beta$ in brain tissue. This is also supported by a recent pathway analysis pinpointing endocytosis and clathrin/AP2-adaptor complex as important mechanisms in dementia [61-63].

Vascular dementia is a disorder in the blood supply of the brain, caused by preceding hemorrhage or ischemia located in either larger vessels or in the microvasculature [64]. Accumulation of amyloid- $\beta$ in the vessel walls of the blood-brain barrier could lead to microinfarcts or microbleeds eventually causing vascular dementia but not necessarily clinically overt stroke. The present findings may emphasize the blood-brain barrier as a delicate border structure between brain and vasculature and supports the now widespread understanding of mixed pathology in most dementia cases. Thus, it is biologically meaningful that disruptions in this pathway affect both brain and vascular disease, in contrast to other dementia susceptibility genes, that exert their effects only in brain $[65,66]$.

An important limitation to this study is that endpoints are based on ICD registry codes from hospitals and death certificates diagnosed in routine clinical practice and thus only captures individuals in contact with hospitals. This is in contrast to research studies where all individuals living in one area are examined and diagnosed with use of standardized instruments, trained staff and standardized diagnostic methods [67]. Consequently, an inherent limitation of using registry-based diagnoses is underdiagnosis. Another limitations is that even though the national Danish registries are regarded among the best of its kind [43, 44], and the quality of the Danish registry-based dementia diagnoses previously has been validated, including a full clinical workup performed by dementia experts [48], the subtypes of dementia, especially vascular dementia and other rare forms, are uncertain [48]. Therefore, we have emphasized throughout that the diagnostic uncertainty should be considered when interpreting the results for vascular dementia. Consequently, we named this subtype "suggested vascular dementia" throughout the paper. Another potential limitation is the uncertainty of age at onset for dementia diseases. It is commonly accepted that for dementia prodromal phases can last for decades. Hence, we cannot exclude that some of the participants receiving a dementia diagnosis during our follow-up time already have dementia pathology at baseline.

Strengths of this study are the prospective design and the large, well-characterized, ethnically homogeneous cohort of the general population with no losses to follow-up. Furthermore, no differences of participant characteristics across weighted/simple allele score groups were observed and therefore obvious confounding of the gene scores could be excluded. Furthermore, there was no interaction between the genetic scores and age at baseline, age at diagnosis, or $A P O E$ genotype. Due to the extensive phenotyping of biochemical and other quantities in the present cohorts, we could also evaluate blood pressure, heart rate, electrolytes, and measures of kidney function, liver function, glucose metabolism and inflammation. We found no significant variation across weighted/simple allele score groups, suggesting that this molecular pathway may be safe to target therapeutically. The sufficient quality of the Danish registry-based all dementia and Alzheimer's disease diagnoses was further supported by the well-known association with the apolipoprotein E $\varepsilon 4$ allele in the present cohorts [42] and by the present metaanalyses for each individual SNP showing similar estimates in our cohorts as those found by the IGAP [16]. Diagnoses of stroke in our cohorts have been validated by trained physicians using the WHO definition of cerebrovascular disease, as previously described [68].

In conclusion, by combining common genetic variation in four genes with possible function in the blood-brain barrier, we found an increased risk of Alzheimer's disease, all dementia and suggested vascular dementia with increasing weighted/simple allele score groups independent of the $A P O E \varepsilon 4$ allele. These findings may suggest that clathrinmediated endocytosis in clearance of amyloid- $\beta$ across the blood-brain barrier is important for the integrity of both brain tissue and cerebral vessels.

Acknowledgements We are indebted to staff and participants of the Copenhagen General Population Study and the Copenhagen City Heart Study for their important contributions to our study. We thank the International Genomics of Alzheimer's Project (IGAP) for providing summary results data for these analyses [16]. The investigators within IGAP contributed to the design and implementation of IGAP and/or provided data but did not participate in analysis or writing of this report. IGAP was made possible by the generous participation of the control subjects, the patients, and their families. The i-Select chips were funded by the French National Foundation on Alzheimer's disease and related disorders. EADI was supported by the LABEX (laboratory of excellence program investment for the future) DISTALZ Grant, Inserm, Institut Pasteur de Lille, Université de Lille 2 and the Lille University Hospital. GERAD was supported by the Medical Research Council (Grant No. 503480), Alzheimer's Research UK (Grant No. 503176), the Wellcome Trust (Grant No. 082604/2/07/Z) and German Federal Ministry of Education and Research (BMBF): Competence Network Dementia (CND) Grant Nos. 01GI0102, 01GI0711, 01GI0420. CHARGE was partly supported by the NIH/NIA Grant R01 AG033193 and the NIA AG081220 and AGES contract N01-AG-12100, the NHLBI Grant R01 HL105756, the Icelandic Heart Association, and the Erasmus Medical 
Center and Erasmus University. ADGC was supported by the NIH/ NIA Grants: U01 AG032984, U24 AG021886, U01 AG016976, and the Alzheimer's Association Grant ADGC-10-196728 [16].

Author contributions IJR: study concept and design, acquisition of data, statistical analysis, analysis and interpretation of data, drafting of the manuscript, critical revision of the manuscript for important intellectual content, final approval for submission. AT-H: acquisition of data, critical revision of the manuscript for important intellectual content, obtained funding, administrative, technical, and material support, final approval for submission. KLR: acquisition of data, statistical analysis, analysis and interpretation of data, critical revision of the manuscript for important intellectual content, final approval for submission. BGN: acquisition of data, critical revision of the manuscript for important intellectual content, obtained funding, administrative, technical, and material support, final approval for submission. RF-S: study concept and design, acquisition of data, statistical analysis, analysis and interpretation of data, drafting of the manuscript, critical revision of the manuscript for important intellectual content, obtained funding, administrative, technical, and material support, study supervision, final approval for submission, accountable for all aspects of the work.

Funding This work was supported by the Danish Medical Research Council (Grant No. 10-081618), by the Research Council at Rigshospitalet (PhD fellowship to IJR), by The Danish Alzheimer Research Foundation, by The Lundbeck Foundation and by M.L. Jørgensen \& Gunnar Hansen's Fund. The funding sources had no role in the design and conduct of the study; collection, management, analysis, and interpretation of the data; preparation, review, or approval of the manuscript; and decision to submit the manuscript for publication.

\section{Compliance with ethical standards}

Conflict of interest The authors declare that they have no conflict of interest.

Availability of data and materials The IGAP dataset is available at http://web.pasteur-lille.fr/en/recherche/u744/igap/igap_download.php. The CCHS and the CGPS datasets are not publicly available due to the Danish law on data protection but are available from the corresponding author on reasonable request.

Ethical approval The Copenhagen studies were approved by institutional review boards and Danish ethical committees [No. (KF) 100.2039/91 and No. (KF) 01-144/01], and were conducted according to the Declaration of Helsinki. Written informed consent was obtained from participants.

Open Access This article is distributed under the terms of the Creative Commons Attribution 4.0 International License (http://creativeco mmons.org/licenses/by/4.0/), which permits unrestricted use, distribution, and reproduction in any medium, provided you give appropriate credit to the original author(s) and the source, provide a link to the Creative Commons license, and indicate if changes were made.

\section{References}

1. World Health Organisation. The epidemiology and impact of dementia: current state and future trends. In: First WHO ministerial conference on global action against dementia; 2015. p. 1-4.
2. Baumgart M, Snyder HM, Carrillo MC, Fazio S, Kim H, Johns H. Summary of the evidence on modifiable risk factors for cognitive decline and dementia: a population-based perspective. Alzheimer's Dement. 2015;11:718-26.

3. Querfurth HW, Laferla FM. Alzheimer's disease. N Engl J Med. 2010;362:329-44.

4. Snowdon D, Greiner L, Mortimer J, Riley K, Greiner P, Markesbery W. Brain infarction and the clinical expression of Alzheimer disease: the Nun Study. JAMA. 1997;277:813-7.

5. Jellinger KA. Prevalence and impact of cerebrovascular lesions in Alzheimer and lewy body diseases. Neurodegener Dis. 2010;7:112-5.

6. Kalaria RN. Vascular basis for brain degeneration: faltering controls and risk factors for dementia. Nutr Rev. 2010;68:1-22.

7. Zlokovic BV. Neurovascular pathways to neurodegeneration in Alzheimer's disease and other disorders. Nat Rev Neurosci. 2011;12:723-38.

8. de la Torre JC. Vascular risk factor detection and control may prevent Alzheimer's disease. Ageing Res Rev. 2010;9:218-25.

9. Hardy J, Allsop D. Amyloid deposition as the central event in the aetiology of Alzheimer's disease. Trends Pharmacol Sci. 1991;12:383-8.

10. Hardy JA, Higgins GA. Alzheimer's disease: the amyloid cascade hypothesis. Science. 1992;256:184-5.

11. Hardy J, Selkoe DJ. The amyloid hypothesis of Alzheimer's disease: progress and problems on the road to therapeutics. Science. 2002;297:353-6.

12. Musiek ES, Holtzman DM. Three dimensions of the amyloid hypothesis: time, space and "wingmen". Nat Neurosci. 2015;18:800-6.

13. Shibata M, Yamada S, Ram Kumar S, Calero M, Bading J, Frangione B, et al. Clearance of Alzheimer's amyloid- $\beta 1-40$ peptide from brain by LDL receptor-related protein-1 at the blood-brain barrier. J Clin Invest. 2000;106:1489-99.

14. Selkoe DJ. Clearing the brain's amyloid cobwebs. Neuron. 2001;32:177-80.

15. Mawuenyega KG, Sigurdson W, Ovod V, Munsell L, Kasten T, Morris JC, et al. Decreased clearance of CNS $\beta$-amyloid in Alzheimer's disease. Science. 2010;330:1774.

16. Lambert JC, Ibrahim-Verbaas CA, Harold D, Naj AC, Sims R, Bellenguez C, et al. Meta-analysis of 74,046 individuals identifies 11 new susceptibility loci for Alzheimer's disease. Nat Genet. 2013;45:1452-8.

17. Dreyling MH, Martinez-Climent JA, Zheng M, Mao J, Rowley JD, Bohlander SK. The $\mathrm{t}(10 ; 11)(\mathrm{p} 13 ; \mathrm{q} 14)$ in the U937 cell line results in the fusion of the AF10 gene and CALM, encoding a new member of the AP-3 clathrin assembly protein family. Proc Natl Acad Sci USA. 1996;93:4804-9.

18. Tebar F, Bohlander SK, Sorkin A. Clathrin assembly lymphoid myeloid leukemia (CALM) protein: localization in endocytic-coated pits, interactions with clathrin, and the impact of overexpression on clathrin-mediated traffic. Mol Biol Cell. 1999; 10:2687-702.

19. Wigge P, Köhler K, Vallis Y, Doyle CA, Owen D, Hunt SP, et al. Amphiphysin heterodimers: potential role in clathrin-mediated endocytosis. Mol Biol Cell. 1997;8:2003-15.

20. Wigge P, McMahon HT. The amphiphysin family of proteins and their role in endocytosis at the synapse. Trends Neurosci. 1998;21:339-44.

21. Ramjaun AR, Micheva KD, Bouchelet I, McPherson PS. Identification and characterization of a nerve terminal-enriched amphiphysin isoform. J Biol Chem. 1997;272:16700-6.

22. Dustin ML, Olszowy MW, Holdorf AD, Li J, Bromley S, Desai N, et al. A novel adaptor protein orchestrates receptor patterning and cytoskeletal polarity in T-cell contacts. Cell. 1998;94:667-77. 
23. Shih NY, Li J, Karpitskii V, Nguyen A, Dustin ML, Kanagawa $\mathrm{O}$, et al. Congenital nephrotic syndrome in mice lacking CD2associated protein. Science. 1999;286:312-5.

24. Kajiho H, Saito K, Tsujita K, Kontani K, Araki Y, Kurosu H, et al. RIN3: a novel Rab5 GEF interacting with amphiphysin II involved in the early endocytic pathway. J Cell Sci. 2003;116:4159-68.

25. Neuvonen M, Kazlauskas A, Martikainen M, Hinkkanen A, Ahola T, Saksela K. SH3 domain-mediated recruitment of host cell amphiphysins by alphavirus nsP3 promotes viral RNA replication. PLoS Pathog. 2011;7:e1002383.

26. Carter C. Alzheimer's disease: APP, gamma secretase, APOE, CLU, CR1, PICALM, ABCA7, BIN1, CD2AP, CD33, EPHA1, and MS4A2, and their relationships with herpes simplex, C. Pneumoniae, other suspect pathogens, and the immune system. Int $\mathbf{J}$ Alzheimers Dis. 2011;2011:501862.

27. Rouka E, Simister PC, Janning M, Kumbrink J, Konstantinou T, Muniz JRC, et al. Differential recognition preferences of the three Src homology 3 (SH3) domains from the adaptor CD2-associated protein (CD2AP) and direct association with Ras and Rab interactor 3 (RIN3). J Biol Chem. 2015;290:25275-92.

28. Treusch S, Hamamichi S, Goodman JL, Matlack KES, Chung $\mathrm{CY}, \mathrm{Baru} \mathrm{V}$, et al. Functional links between A $\beta$ toxicity, endocytic trafficking, and Alzheimer's disease risk factors in yeast. Science. 2011;334:1241-5.

29. Zhao Z, Sagare AP, Ma Q, Halliday MR, Kong P, Kisler K, et al. Central role for PICALM in amyloid- $\beta$ blood-brain barrier transcytosis and clearance. Nat Neurosci. 2015;18:978-87.

30. Harold D, Abraham R, Hollingworth P, Sims R, Gerrish A, Hamshere $\mathrm{M}$, et al. Genome-wide association study identifies variants at CLU and PICALM associated with Alzheimer's disease, and shows evidence for additional susceptibility genes. Nat Genet. 2009;41:1088-93.

31. Hollingworth P, Harold D, Sims R, Gerrish A, Lambert J-C, Carrasquillo MM, et al. Common variants at ABCA7, MS4A6A/ MS4A4E, EPHA1, CD33 and CD2AP are associated with Alzheimer's disease. Nat Genet. 2011;43:429-35.

32. Tan M-S, Yu J-T, Tan L. Bridging integrator 1 (BIN1): form, function, and Alzheimer's disease. Trends Mol Med. 2013;19:594-603.

33. Beecham GW, Hamilton K, Naj AC, Martin ER, Huentelman M, Myers AJ, et al. Genome-wide association meta-analysis of neuropathologic features of Alzheimer's disease and related dementias. PLoS Genet. 2014;10:e1004606.

34. Seshadri S, Fitzpatrick AL, Ikram A, DeStafano AL, Gudnason V, Boada M, et al. Genome-wide analysis of genetic loci associated with Alzheimer disease. J Am Med Assoc. 2010;303:1832-40.

35. Lambert J-C, Heath S, Even G, Campion D, Sleegers K, Hiltunen $\mathrm{M}$, et al. Genome-wide association study identifies variants at CLU and CR1 associated with Alzheimer's disease. Nat Genet. 2009;41:1094-9.

36. Corder EH, Saunders AM, Strittmatter WJ, Schmechel DE, Gaskell PC, Small W, et al. Gene dose of apolipoprotein E type 4 allele and the risk of Alzheimer's disease in late onset families. Science. 1993;261:921-3.

37. Naj AC, Jun G, Beecham GW, Wang L, Vardarajan BN, Buros J, et al. Common variants at MS4A4/MS4A6E, CD2AP, CD33 and EPHA1 are associated with late-onset Alzheimer's disease. Nat Genet. 2011;43:436-41.

38. Miller SE, Sahlender DA, Graham SC, Höning S, Robinson MS, Peden AA, et al. The molecular basis for the endocytosis of small R-SNAREs by the clathrin adaptor CALM. Cell. 2011;147:1118-31.

39. Bell RD, Winkler EA, Singh I, Sagare AP, Deane R, Wu Z, et al. Apolipoprotein E controls cerebrovascular integrity via cyclophilin A. Nature. 2012;485:512-6.
40. Frikke-Schmidt R, Nordestgaard BG, Stene MCA, Sethi AA, Remaley AT, Schnohr P, et al. Association of loss-of-function mutations in the ABCA1 gene with high-density lipoprotein cholesterol levels and risk of ischemic heart disease. JAMA. 2008;299:2524-32.

41. Jørgensen AB, Frikke-Schmidt R, Nordestgaard BG, TybjærgHansen A. Loss-of-function mutations in APOC3 and risk of ischemic vascular disease. N Engl J Med. 2014;371:32-41.

42. Rasmussen KL, Tybjærg-Hansen A, Nordestgaard BG, FrikkeSchmidt R. Plasma levels of apolipoprotein E and risk of dementia in the general population. Ann Neurol. 2015;77:301-11.

43. Lynge E, Sandegaard JL, Rebolj M. The Danish national patient register. Scand J Public Health. 2011;39:30-3.

44. Helweg-Larsen K. The Danish register of causes of death. Scand J Public Health. 2011;39:26-9.

45. Rasmussen KL, Tybjærg-Hansen A, Nordestgaard BG, FrikkeSchmidt R. Plasma apolipoprotein E levels and risk of dementia: a Mendelian randomization study of 106,562 individuals. Alzheimer's Dement. 2018;14:71-80.

46. Mckhann G, Drachman D, Folstein M, Katzman R, Price D, Stadlan EM. Clinical diagnosis of Alzheimer's disease: report of the NINCDS-ADRDA Work Group under the auspices of Department of Health and Human Services Task Force on Alzheimer's Disease. Neurology. 1984;34:939-44.

47. McKhann GM, Knopman DS, Chertkow H, Hyman BT, Jack CR, Kawas $\mathrm{CH}$, et al. The diagnosis of dementia due to Alzheimer's disease: recommendations from the National Institute on AgingAlzheimer's Association workgroups on diagnostic guidelines for Alzheimer's disease. Alzheimer's Dement. 2011;7:263-9.

48. Phung TKT, Andersen BB, Høgh P, Kessing LV, Mortensen PB, Waldemar G. Validity of dementia diagnoses in the danish hospital registers. Dement Geriatr Cogn Disord. 2007;24:220-8.

49. Román GC, Tatemichi TK, Erkinjuntti T, Cummings JL, Masdeu JC, Garcia JH, et al. Vascular dementia: diagnostic criteria for research studies: report of the NINDS-AIREN International Workshop. Neurology. 1993;43:250-60.

50. Sachdev P, Kalaria R, O’Brien J, Skoog I, Alladi S, Black SE, et al. Diagnostic criteria for vascular cognitive disorders. Alzheimer Dis Assoc Disord. 2014;28:206-18.

51. Friedewald WT, Levy RI, Fredrickson DS. Estimation of the concentration of low-density lipoprotein cholesterol in plasma, without use of the preparative ultracentrifuge. Clin Chem. 1972;18:499-502.

52. Kidney Disease: Improving Global Outcomes (KDIGO) CKD Group. KDIGO clinical practice guideline for the evaluation and management of cronic kidney disease. Kidney Int Suppl. 2013;3:1-150.

53. Purcell SM, Wray NR, Stone JL, Visscher PM, O'Donovan MC, Sullivan $\mathrm{PF}$, et al. Common polygenic variation contributes to risk of schizophrenia and bipolar disorder. Nature. 2009;460:748-52.

54. Fine JP, Gray RJ. A proportional hazards model for the subdistribution of a competing risk. J Am Stat Assoc. 1999;94:496-509.

55. Bucci C, Parton RG, Mather IH, Stunnenberg H, Simons K, Hoflack B, et al. The small GTPase rab5 functions as a regulatory factor in the early endocytic pathway. Cell. 1992;70:715-28.

56. Gorvel JP, Chavrier P, Zerial M, Gruenberg J. Rab5 controls early endosome fusion in vitro. Cell. 1991;64:915-25.

57. Zeigerer A, Gilleron J, Bogorad RL, Marsico G, Nonaka H, Seifert $\mathrm{S}$, et al. Rab5 is necessary for the biogenesis of the endolysosomal system in vivo. Nature. 2012;485:465-70.

58. Ullrich O, Reinsch S, Urbé S, Zerial M, Parton RG. Rab11 regulates recycling through the pericentriolar recycling endosome. J Cell Biol. 1996;135:913-24.

59. Takahashi S, Kubo K, Waguri S, Yabashi A, Shin H-W, Katoh $\mathrm{Y}$, et al. Rab11 regulates exocytosis of recycling vesicles at the plasma membrane. J Cell Sci. 2012;125:4049-57. 
60. Yui N, Lu HAJ, Chen Y, Nomura N, Bouley R, Brown D. Basolateral targeting and microtubule-dependent transcytosis of the aquaporin-2 water channel. AJP Cell Physiol. 2013;304:C38-148.

61. Ahmad S, Bannister C, van der Lee SJ, Vojinovic D, Adams HHH, Ramirez A, et al. Disentangling the biological pathways involved in early features of Alzheimer's disease in the Rotterdam Study. Alzheimer's Dement. 2018;14:1-10.

62. Guerreiro R, Brás J, Hardy J. XSnapShot: genetics of Alzheimer's disease. Cell. 2013;155(968-968):e1.

63. Jones L, Lambert JC, Wang LS, Choi SH, Harold D, Vedernikov A, et al. Convergent genetic and expression data implicate immunity in Alzheimer's disease. Alzheimer's Dement. 2015;11:658-71.

64. Iadecola C. The pathobiology of vascular dementia. Neuron. 2013;80:844-66.

65. Kjeldsen EW, Tybjærg-Hansen A, Nordestgaard BG, FrikkeSchmidt R. ABCA7 and risk of dementia and vascular disease in the Danish population. Ann Clin Transl Neurol. 2018;5:41-51.
66. Nordestgaard LT, Tybjaerg-Hansen A, Rasmussen KL, Nordestgaard BG, Frikke-Schmidt R. Genetic variation in clusterin and risk of dementia and ischemic vascular disease in the general population: cohort studies and meta-analyses of 362,338 individuals. BMC Med. 2018;16:39.

67. Hofman A, Brusselle GGO, Murad SD, Klaver CCW, Nijsten TEC, Peeters RP, et al. The Rotterdam Study: 2016 objectives and design update. Eur J Epidemiol. 2016;30:661-708.

68. Nordestgaard LT, Tybjaerg-Hansen A, Nordestgaard BG, FrikkeSchmidt R. Loss-of-function mutation in ABCA1 and risk of Alzheimer's disease and cerebrovascular disease. Alzheimer's Dement. 2015;11:1430-8.

Publisher's Note Springer Nature remains neutral with regard to jurisdictional claims in published maps and institutional affiliations. 